\title{
Pore segmentation of cement-based materials from backscattered electron images
}

\author{
H.S. Wong ${ }^{1}$, M.K. Head, N.R. Buenfeld \\ Department of Civil and Environmental Engineering, Imperial College London, Imperial College Road, London, \\ SW7 2AZ, UK
}

\begin{abstract}
A technique to segment pores from a normal backscattered electron (BSE) image of cement-based materials is presented. The upper threshold grey level for porosity is determined from the inflection point of the cumulative brightness histogram of the BSE image. This represents a critical point where a small incremental grey value will cause a sudden increase in thresholded area, a condition termed as overflow. The proposed technique was found to be more consistent and reliable than existing methods. Significantly fewer images are required to achieve a satisfactory level of statistical confidence for quantifying porosity.
\end{abstract}

Keywords: Backscattered electron imaging (B), Image analysis (B), Microstructure (B), SEM (B), Porosity

\section{Introduction}

Ever since pioneering work by Scrivener and Pratt [1], backscattered electron (BSE) imaging has been widely used as a technique to examine the microstructure of cement based materials. In a BSE image of a flat-polished section, the backscatter coefficient increases monotonically with the increase in mean atomic number of each phase [2], hence allowing individual phases to be differentiated and quantified using image analysis. In cement and concrete research, BSE imaging has been applied to quantify phases in anhydrous cement grains [3], hydrated cement pastes [4, 5], microstructural gradients in the interfacial transition zone [6, 7], cracks [8] and pore structure $[9,10]$.

Quantification of features via image analysis requires, firstly, good specimen preparation and imaging technique to produce representative images, and secondly, a feature-segmentation algorithm that is objective, precise and reproducible. When features can be segmented with accuracy and consistency, only then meaningful quantitative data can be obtained, that can be used for comparative studies and/or to formulate structure-property relationships. Since individual phases in a BSE image have different brightness intensities, the obvious way to segment features is by

\footnotetext{
${ }^{*}$ Corresponding author. Tel: +44-207-594-5957; Fax: +44-207-225-2716

E-mail address: hong.wong@imperial.ac.uk
} 
grey level thresholding. Ideally, the grey level histogram would be composed of separate peaks corresponding to distinct phases with heights proportional to the relative fractions of each phase. However, as the size of a pixel in a digital image is finite and because of sampling volume effects, the brightness of each pixel does not necessarily represent a single phase alone. Pixels lying on inter-phase boundaries will display an intermediate grey level that averages those of the two sampled phases. This will have an effect of broadening the histogram peaks and, depending on the degree of overlap between the broadened signals, it becomes increasingly difficult to ascertain the appropriate thresholds for feature segmentation.

In cement-based materials, the backscatter coefficient for several hydration products (C-S-H, ettringite, monosulphate) are too close to be individually distinguished on the brightness histogram; a single broad peak is usually observed in the histogram. Pores ${ }^{1}$ are perhaps easiest to segment because the backscatter coefficient of epoxy is substantially smaller than other phases. For example, the backscatter coefficient for araldite $\left(\mathrm{C}_{10} \mathrm{H}_{18} \mathrm{O}_{4}\right)$ is about 0.07 (calculated after Reuter [11]) compared to 0.12 to 0.19 for major hydration products and anhydrous cement phases [12]. The lower threshold level for pores can be set to zero (black pixels) and the segmentation process is then reduced to only determining the upper threshold level. However, problems in defining the exact boundary between pores and the surrounding hydrated paste still exist. Apart from the inherent weaknesses associated with the imaging process, the pore boundary is indistinct due to the diffuse morphology of the C-S-H gel $[5,13]$. Also, a separate pore peak does not always occur in the brightness histogram, particularly when the porosity is low $[5,13]$.

Commonly used methods to select the upper threshold for pores include manual thresholding, the tangent-slope method and entropy maximisation. In manual thresholding, the operator iteratively selects the threshold value so that the segmented pixels correspond satisfactorily to the features of interest in the original image. This is highly subjective, inconsistent between different operators or even the same operator over a period of time or over a range of different samples. It is also affected by operator fatigue. Scrivener et al. [5] found that the grey level at which the tangent to the upper portion of the hydration products (HP) peak intersected the initial tangent on the grey level histogram gave consistent results and was closest to the manual threshold. However, the tangent-slope threshold is difficult to ascertain if a pore peak exists in the histogram for a highly porous sample. In this case, the minimum point between the pore peak and the HP peak can be used for thresholding. This is expected to give the smallest error because it corresponds to the value that affects the fewest pixels [14]. Entropy maximisation is a classical thresholding method based on measuring the information content (entropy) of an image; a detailed explanation of this technique will not be provided, but is

\footnotetext{
${ }^{1}$ In this paper, the term 'pores' is used as a general term for epoxy-filled voids in the paste; this includes capillary
} pores, cracks and hollow hydration shells. 
available elsewhere [15-17]. This method has been used to segment microcracks and voids that are highly contrasted by impregnation with fluorescent dye [18, 19] and Wood's metal [20].

\section{Resolution, brightness and contrast}

A high quality original image is the prerequisite for accurate segmentation of features and subsequent quantification steps. For optimum performance, the electron microscope operating configuration (accelerating voltage, beam current and working distance) must be set depending on the particular contrast produced by the specimen/detector system. The threshold equation [21] can be used to determine the minimum beam current for a required level of contrast so that the smallest probe size is attained. In most cases, however, spatial resolution is limited by the sampling volume from which the signal used for image formation is generated, whose effective size can be substantially larger than the electron beam. In a digital image, pixel spacing also affects resolution and should ideally be about the same as the lateral dimension of the sampling volume.

Some misinterpretation of BSE images arises from a lack of understanding of the need for a bright and properly contrasted image. Inexperienced operators tend to 'enhance' the image during capture by increasing its contrast. However, if contrast is too high, image detail will be lost in the low and high regions of the greyscale, creating a distorted histogram with artificial peaks at both ends of the greyscale. If all the phases are to be quantified, then ideally, the histogram should spread out as much as possible, but not beyond the greyscale spectrum. If the image is initially captured at too high contrast, it is meaningless to reduce the contrast later by image processing, as this will only compress the histogram, but not remove the artificial peaks. Any signal processing performed after image capture, although possibly improving visual appearance, does not increase the information content of the image.

\section{Development of a pore segmentation method}

Fig. 1 shows two BSE images of different mortars taken at 500x magnification. Aggregates were removed from the original image and the brightness histogram of the remaining paste region is displayed. For the mortar with high w/c ratio, the brightness histogram consists of four distinct peaks representing pores, hydration products (HP), calcium hydroxide $(\mathrm{CH})$ and anhydrous cement $(\mathrm{AH})$ phases. For the mortar with low w/c ratio, only two peaks are visible; the larger peak is possibly made up of pixels from HP and $\mathrm{CH}$ phases, while the smaller peak from unreacted cement grains. There is no visible pore peak apparently due to the low volume of pores in the sample. This observation 
suggests that a general segmentation method cannot only depend on the existence of feature peaks on the brightness histogram.

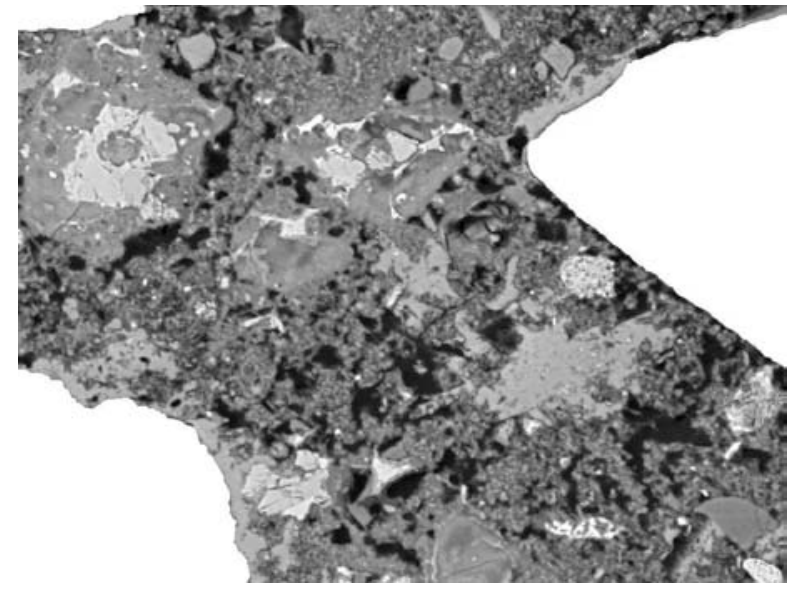

a) Mortar A (w/c: 0.70)

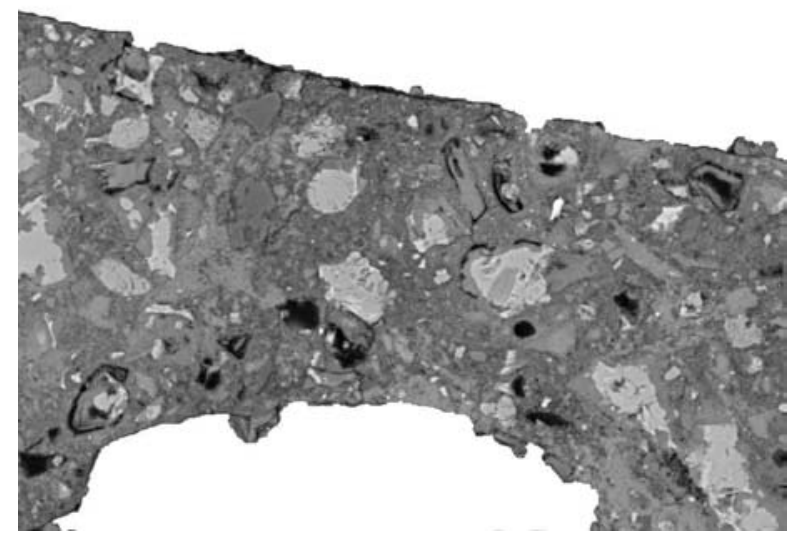

c) Mortar B (w/c: 0.35)

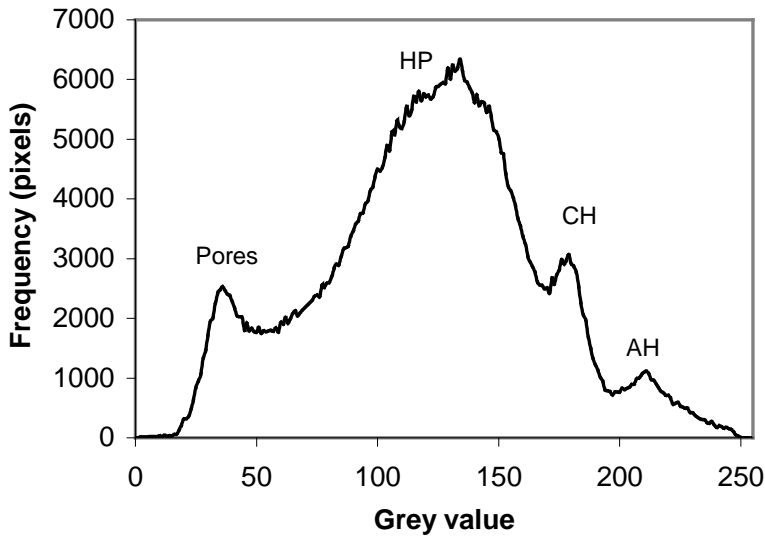

b) Brightness histogram for Mortar A

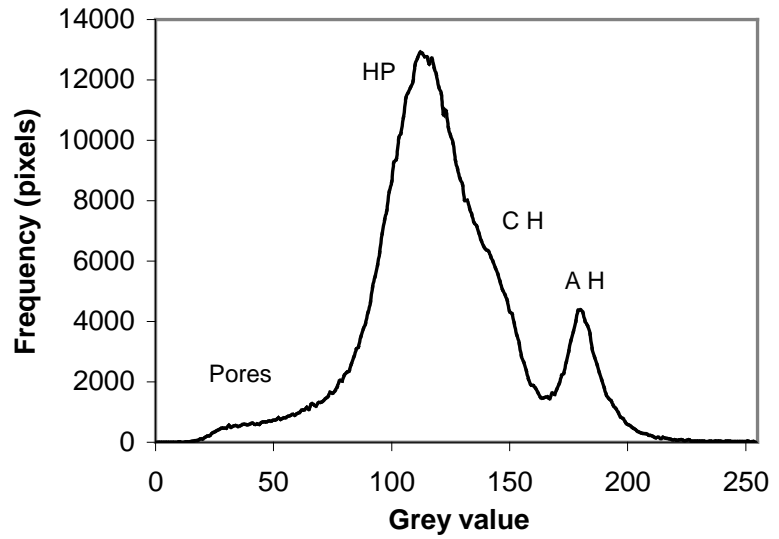

d) Brightness histogram for Mortar B

Fig. 1 BSE image of paste regions from two different mortars at 28 days. The brightness histogram for the low w/c ratio mortar did not show a distinct peak for pores. Field of view is $267 \mu \mathrm{m} \times 200 \mu \mathrm{m}$.

A single capillary pore extracted from Fig. 1(c) is shown in Fig. 2. The change in brightness along the midhorizontal line indicates a gradual drop in grey value from approximately 100 to 20 over a distance of approximately $3 \mu \mathrm{m}$ near the pore boundary. Although the boundary may be atomically sharp, the gradual transition of the measured signal is a result of overlapping sampling volume at the boundary. This is schematically illustrated in Fig. 3. It can also be seen that this effect increases when the boundary is inclined towards the lower atomic number (Z) material (case b), for example, in the case of a shallow pore. The slightly higher signal recorded near the boundary of the high-Z material is due to edge effect. The gradual drop in brightness near the boundary creates uncertainty regarding the true position of the pore edge. One may assume the pore threshold level to be either the upper, middle or lower end of the 'transition 
region'; in each case the measured pore size will be significantly different. However, as observed in Fig, 3, the grey value at the upper end of the transition region provides a closer estimate for the pore threshold.

a)
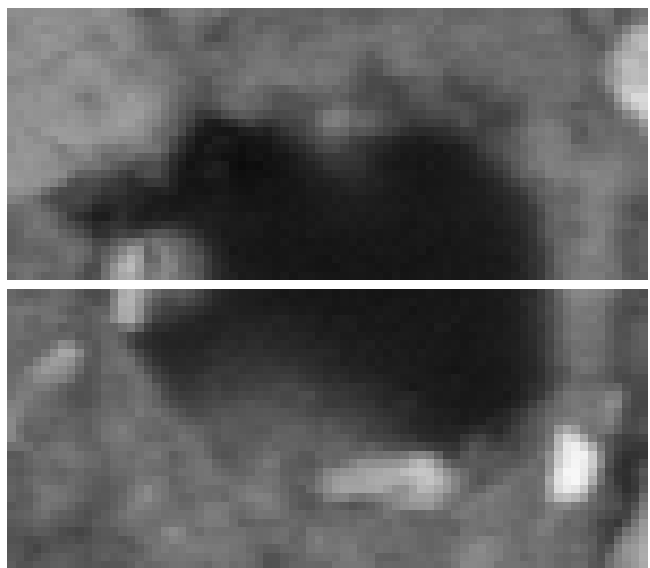

b)

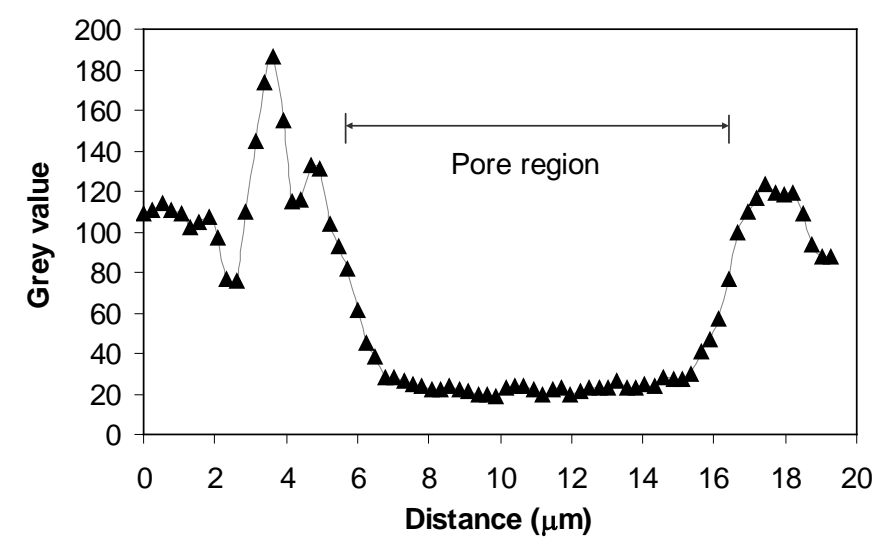

Fig. 2 a) BSE image of a single capillary pore and b) variation of grey value along the horizontal white line. Field of view of the image is approximately $20 \mu \mathrm{m} \times 18 \mu \mathrm{m}$.

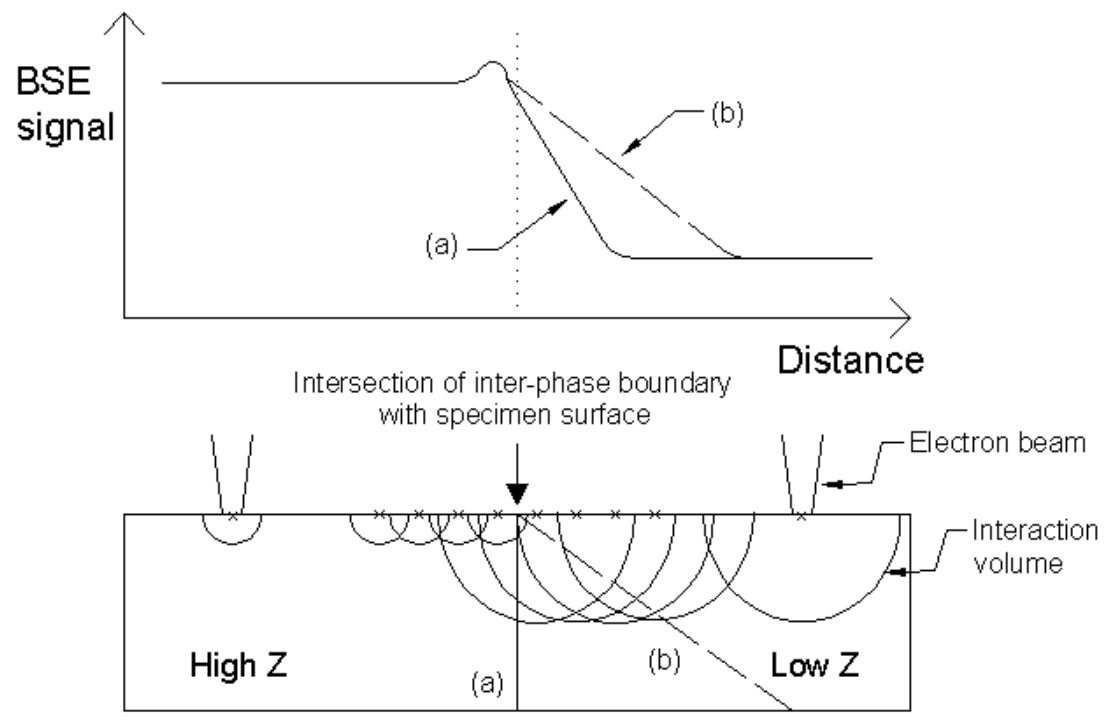

Fig. 3 Schematic illustrating the influence of beam interaction volume on the BSE signal near the inter-phase boundary of a high and a low atomic number ( $\mathrm{Z}$ ) material when the hypothetical boundary is a) perpendicular and $\mathrm{b}$ ) inclined to the specimen surface. (Adapted from Goldstein et al. [21])

Fig. 4 shows a series of images of the same capillary pore with area segmented (white pixels) at different threshold levels. As the threshold is increased, pixels located in the pore centre are segmented first followed by the pixels near the pore boundary. This figure demonstrates the ambiguity of the manual segmentation technique since the operator may select anywhere between 60 and 100 as the threshold. However, between 80 and 110, a sudden increase in area segmented can be observed when the surrounding solid phase is also selected. This is analogous to filling up a pore 
with a fluid. If viewed in plan, the area filled with the fluid will increase slowly as more volume is poured. As it arrives near the rim, a critical point is reached when the liquid will overflow to the surrounding areas and this will lead to a sudden increase of the area covered with the fluid. Therefore, the critical point where the area segmented starts to 'overflow' can provide a good estimate for the pore threshold level.

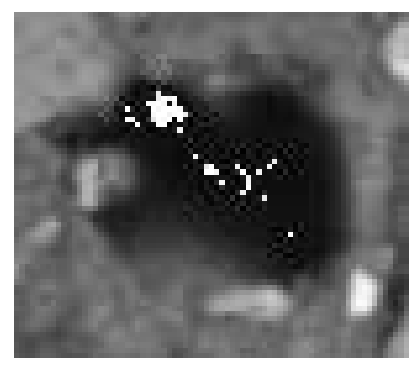

Threshold $=20$

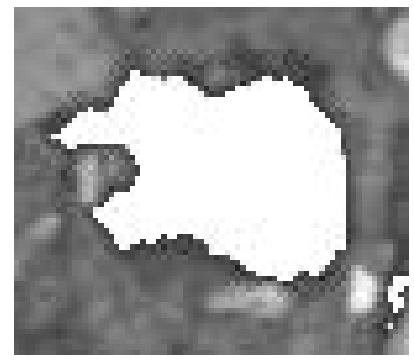

Threshold $=60$

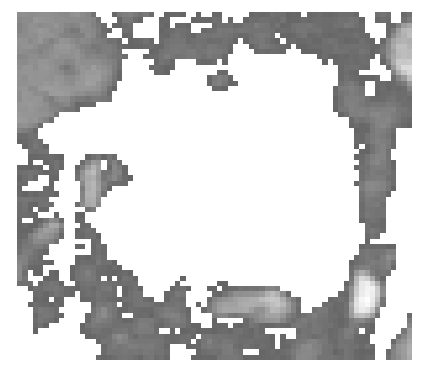

Threshold $=100$

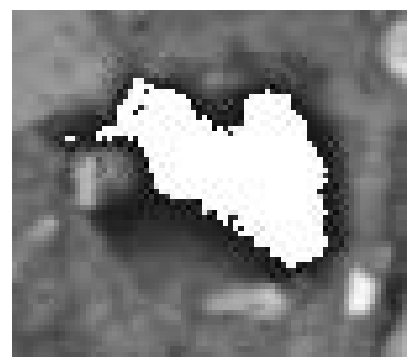

Threshold $=30$

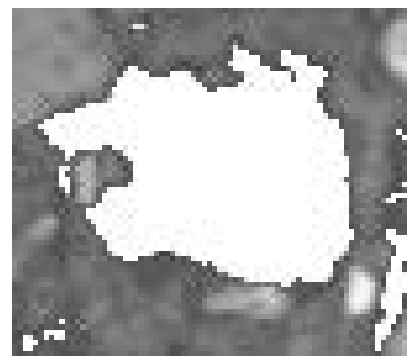

Threshold $=80$

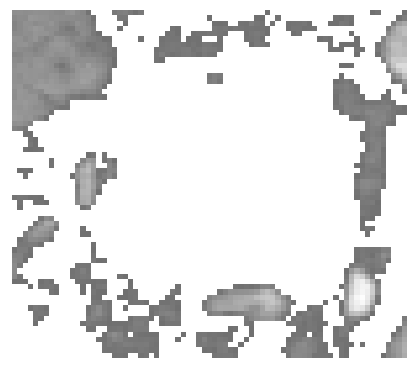

Threshold $=110$

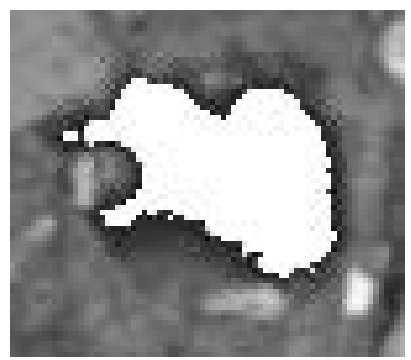

Threshold $=40$

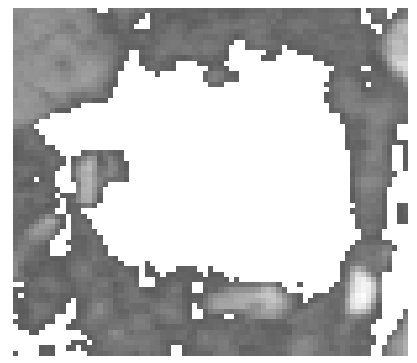

Threshold $=90$

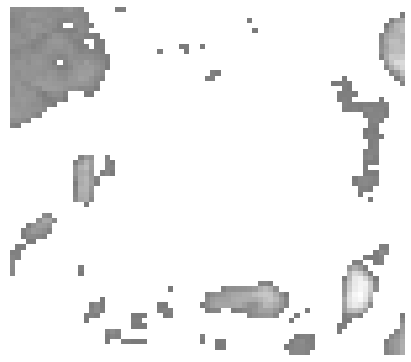

Threshold $=120$

Fig. 4 Change in area segmented (white pixels) at different threshold levels. Between thresholds 80 and 110 , a sudden increase in segmented area is observed when the surrounding paste is also selected.

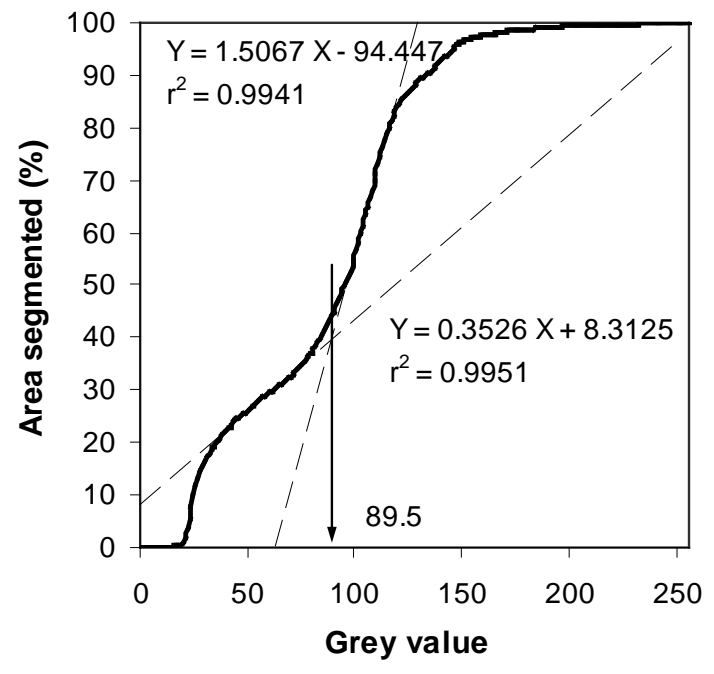


Fig. 5 Change in area segmented with grey value for the pore in Fig. 2. The threshold value for porosity can be estimated from the inflection point.

When the total area segmented is plotted against the threshold level in Fig. 5, the critical overflow point corresponds to the inflection of the cumulative curve, which can be estimated from the intersection between the two linear segments as shown in the figure. The grey value at this intersection can be used as the upper threshold level for porosity. However, this value may slightly overestimate the true overflowing point. A more conservative estimate may be obtained from the point where the curve begins to deviate from the first linear segment. This can be manually selected or approximated by multiplying the grey value obtained at the intersection with a factor of say, 0.9. Application of the proposed overflow criteria for pore segmentation is demonstrated on the full BSE image in Fig. 6.

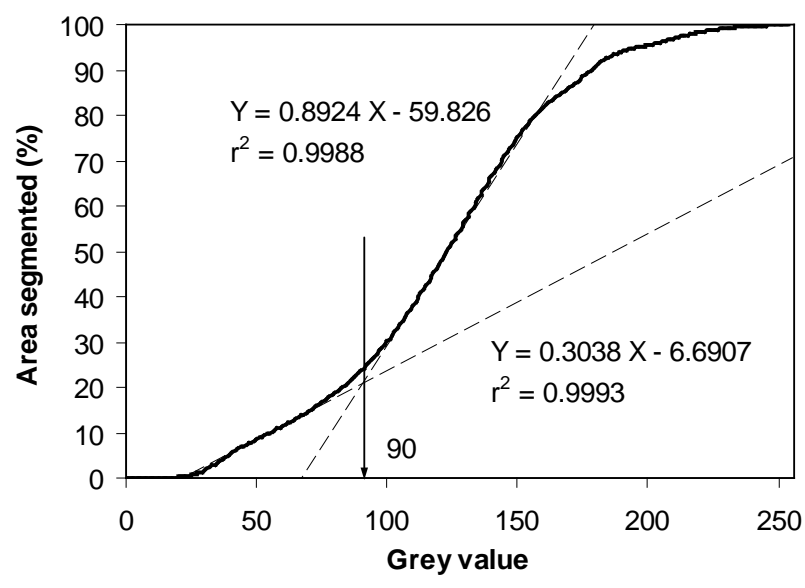

a) Cumulative greyscale histogram for Mortar A

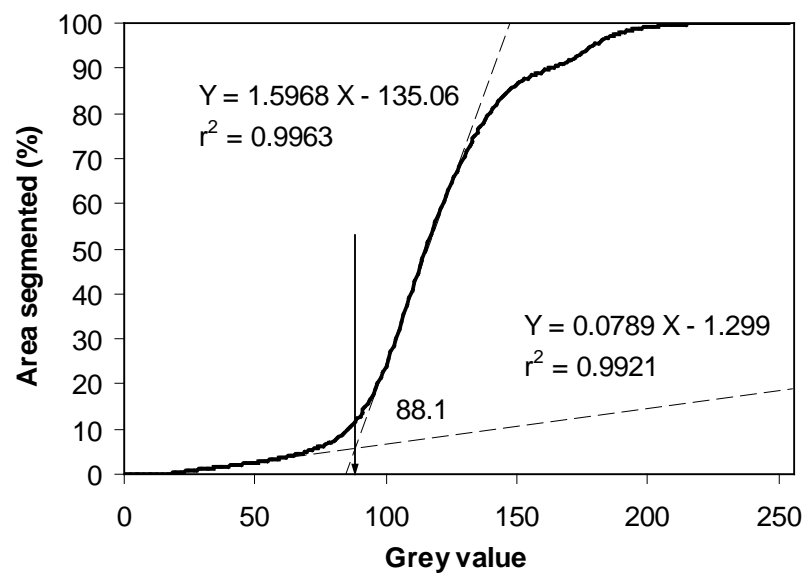

c) Cumulative greyscale histogram for Mortar B

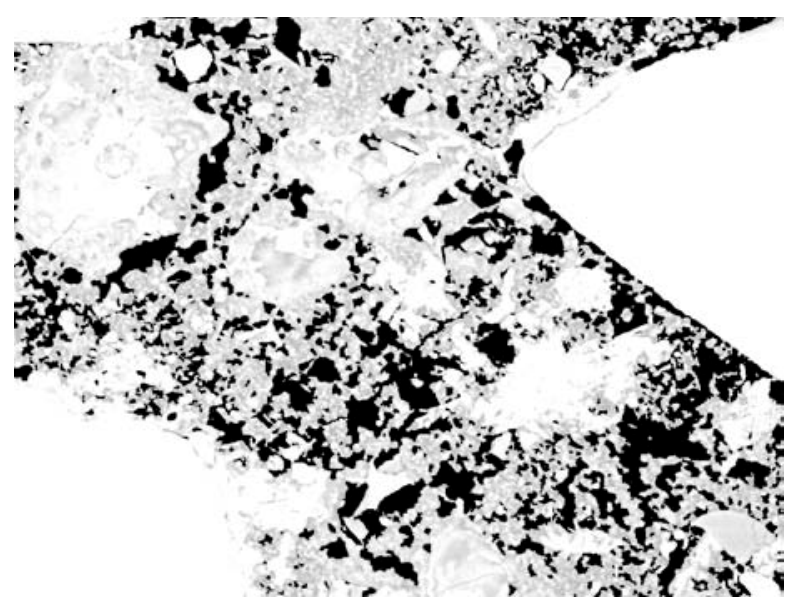

b) Pores segmented (black pixels) from Mortar $\mathrm{A}$ at threshold level 90. Porosity of paste $=23.6 \%$

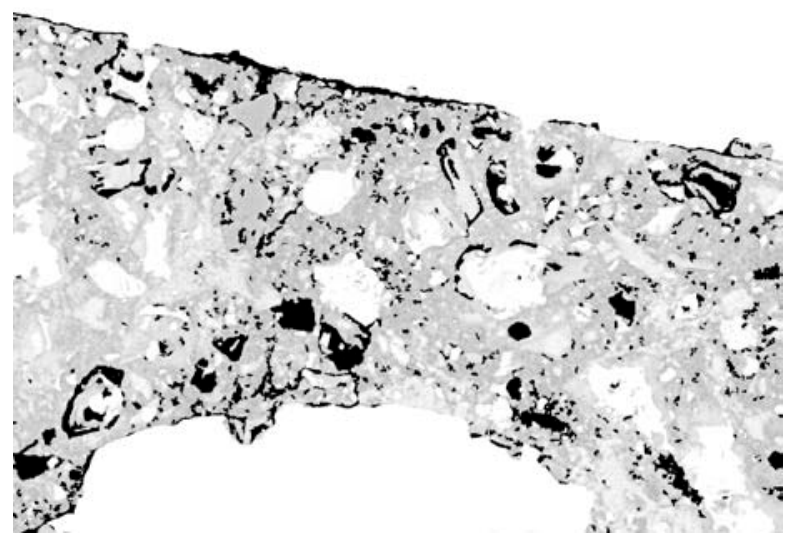

d) Pores segmented (black pixels) from Mortar B at threshold level 88 . Porosity of paste $=11.5 \%$

Fig. 6 Application of the overflow criteria to determine a global threshold level for porosity 


\section{Experimental}

The proposed overflow method and several other existing pore segmentation methods were tested on mortars of w/c ratios 0.35 and 0.70 . OPC and siliceous sand were used to prepare the mortars according to the proportions shown in Table 1. A naphthalene sulphonated type superplasticizer was used for Mortar B at a dosage of $0.5 \%$ by weight of cement. Cylindrical specimens $(100 \phi$ x 250mm) were cast, demoulded after 24 hours, wrapped in cling film and stored at $20^{\circ} \mathrm{C}$. After curing for 28 days, a $10 \mathrm{~mm}$ thick disc was cut from each cylinder at approximately $100 \mathrm{~mm}$ from the bottom cast face, from which a block specimen (40 x 20 x 10mm) for microscopy was prepared. The block sample was dried in an oven at $50^{\circ} \mathrm{C}$ for 3 days, epoxy impregnated, polished using diamond paste down to $1 / 4 \mu \mathrm{m}$ and coated with carbon. A non-aqueous solution was used as lubricant for cutting and polishing. Acetone was used as cleaning fluid.

Table 1 Mixture proportions

\begin{tabular}{cccc}
\hline Mixture & Cement $\left(\mathrm{kg} / \mathrm{m}^{3}\right)$ & Sand $\left(\mathrm{kg} / \mathrm{m}^{3}\right)$ & w/c ratio \\
\hline Mortar A & 365 & 1635 & 0.70 \\
Mortar B & 550 & 1635 & 0.35 \\
\hline
\end{tabular}

A JEOL 5410LV scanning electron microscope equipped with a backscatter electron detector was used for imaging. The microscope was operated at $20 \mathrm{kV}$ accelerating voltage and $15 \mathrm{~mm}$ working distance. Thirty greyscale images were collected per sample at 500x magnification. Images were digitised to 1024 x 768 pixels at a pixel spacing of $0.26 \mu \mathrm{m}$. In order to ensure random sampling, the microscope stage was programmed to move in a grid fashion, stopping at thirty predefined, equally spaced coordinates. Sections near the specimen edge were not imaged to avoid areas that may have been saw-damaged. During image capture, the brightness and contrast setting was adjusted such that ten bins from each end of the greyscale were left empty, i.e. no pixel count is registered on the 0-10 and 245-255 grey values of the captured image. This was done to ensure that the brightness histogram is well spread out but does not exceed the greyscale spectrum. It also indirectly calibrates the microscope so that the brightness and contrast for each frame is relatively the same. 


\section{Image Analysis}

Image analysis was performed using AnalySIS software developed by Soft Imaging System GmbH. Aggregate particles were first removed from the original image by a combined grey level thresholding, filtering and binary operation [22]. Various methods for pore segmentation were tested. Manual thresholding was done by the same operator (HSW) and prior to the other methods to minimise bias. An initial threshold corresponding to larger pores was first set by judgement. The pixels selected were colour coded and superimposed on to the original image to check if it matched the pores in the original image. This visual comparison was made at higher magnification to reveal smaller pores and detailed pore boundary; the threshold was then adjusted until all pores were satisfactory covered by the coloured phase. The tangent-slope thresholding method was done according to Scrivener et al. [5]. For mortar A, where a pore peak existed in the histogram, the minimum point between the pore peak and the HP peak was taken as the porosity threshold. This can be located by referring to the first-derivation curve of the brightness histogram. Thresholding by entropy maximisation was done according to Kapur et al. [16] (Appendix I).

For the proposed method, two values were tested: Overflow 1 is the grey value at the inflection point and Overflow 2 is a more conservative estimate obtained by factoring Overflow 1 by 0.9. Another method tested is the autothreshold utility that is available in the image analysis software. This method calculates thresholds based on the overall histogram of the image. A priori knowledge of the number of phases that exist in the image is required. In this study, the greyscale histogram after having the aggregate particles removed was assumed to consist of four phases: pores, $\mathrm{HP}, \mathrm{CH}$ and anhydrous products. Thresholds are calculated using a statistical comparison of the grey values of the various parts of the image. The algorithm first selects a grey value randomly as threshold for each phase. The average grey value and the standard deviation are then calculated for the grey value ranges both to the left and the right of this initial threshold. The system will then continue to adjust the randomly selected threshold until optimal averages and standard deviations have been attained.

Once the various pore thresholds had been determined, binary segmentation was performed on the images to separate pores from other phases. Based on principles of stereology [23], the volume fraction of a particular phase in an isotropic material is equivalent to its area fraction observed on a random plane-section. Porosity was then calculated as a percentage of the segmented pore area to the total paste area. However, features smaller than 10 pixels were regarded as noise and excluded from the porosity calculation. This sets a lower size limit of the detected pores to 10 pixels, which have an equivalent circular diameter of $0.9 \mu \mathrm{m}$. 


\section{Results and Discussion}

Table 2 shows results of the average porosity $\mu$ and standard deviation $S$, after processing all thirty images using various segmentation methods. The minimum number of frames required to give a level of statistical confidence is also provided. The number of frames required per sample, $N$, such that the sample mean is within $10 \%$ of the true population mean $\mu$, at 95\% degree of confidence is calculated from Student's t-distribution (Appendix II).

Entropy maximisation produced the most consistent thresholded porosity since only one frame was required to achieve a statistically significant result, but gave unrealistically high porosity values for both mortars. This method may only be suitable when the pores are highly contrasted. The auto-detection (AnalySIS) method performed well for Mortar A, but also returned a high porosity value for Mortar B. The proposed overflow technique was found to perform better than other methods based on the coefficient of variation and the number of frames required to achieve a desired degree of confidence. It is worthwhile to note here that the two linear segments used to determine the critical overflow point can be found in each of the analysed images. For Overflow 1, less than 15 frames were needed compared to 30-100 frames for other methods. This highlights the importance of using an objective and reproducible threshold selection criteria. Near the critical overflow point, any subtle changes in the threshold value will create an enormous response in the total thresholded area. Hence, manual selection of the threshold value will most certainly lead to a high variance in the segmented porosity.

Table 2 Statistical analyses

\begin{tabular}{|c|c|c|c|c|c|c|c|c|}
\hline \multirow{2}{*}{ Segmentation method } & \multicolumn{4}{|c|}{ Mortar A (w/c: 0.70) } & \multicolumn{4}{|c|}{ Mortar B (w/c: 0.35) } \\
\hline & $\mu(\%)$ & $\mathrm{S}(\%)$ & $(S / \mu) \times 100 \%$ & $\mathrm{~N}$ & $\mu(\%)$ & S (\%) & $(S / \mu) \times 100 \%$ & $\mathrm{~N}$ \\
\hline 1. Manual & 22.0 & 7.3 & 33.3 & 46 & 11.3 & 5.6 & 49.5 & 103 \\
\hline $\begin{array}{l}\text { 2. Minimum-point between } \\
\text { peaks }\end{array}$ & 13.2 & 5.0 & 37.8 & 60 & - & - & - & - \\
\hline 3. Tangent-slope & - & - & - & - & 6.3 & 2.4 & 37.6 & 59 \\
\hline 4. Entropy maximisation & 47.9 & 0.7 & 1.4 & 1 & 48.1 & 1.2 & 2.4 & 1 \\
\hline 5. Auto (AnalySIS) & 23.4 & 3.3 & 14.0 & 8 & 21.5 & 5.8 & 26.8 & 30 \\
\hline 6. Overflow (1) & 21.2 & 3.8 & 17.7 & 13 & 10.6 & 1.5 & 14.2 & 8 \\
\hline 7. Overflow (2) & 15.9 & 4.2 & 26.6 & 30 & 6.7 & 1.4 & 21.9 & 20 \\
\hline
\end{tabular}

In Fig. 7, the cumulative average porosity (Appendix III) is plotted against the number of frames. This value is different from the average porosity given in Table 2. Since the distribution of pores in hydrated cement paste is not homogenous, there exists a high variability in pores from frame to frame. The cumulative average porosity after frame $\mathrm{i}$ gives a pore fraction value that is measured over a larger combined area. The figure shows that the cumulative average 
porosity obtained from the overflow criteria stabilises after about 15 frames. This remains valid even when the order of the images is shuffled. It is also interesting to note that the manually thresholded porosity matches with Overflow 1 values and the tangent-slope values with Overflow 2. The manually thresholded porosity is slightly higher than Overflow 1, possibly because human bias tends to slightly overestimate the area of the features of interest [14]. When visually selecting a threshold, the operator is more tolerant of settings that include additional pixels from background region (solid) along with the features (pores), than they are at settings that exclude some pixels from the background.

a)

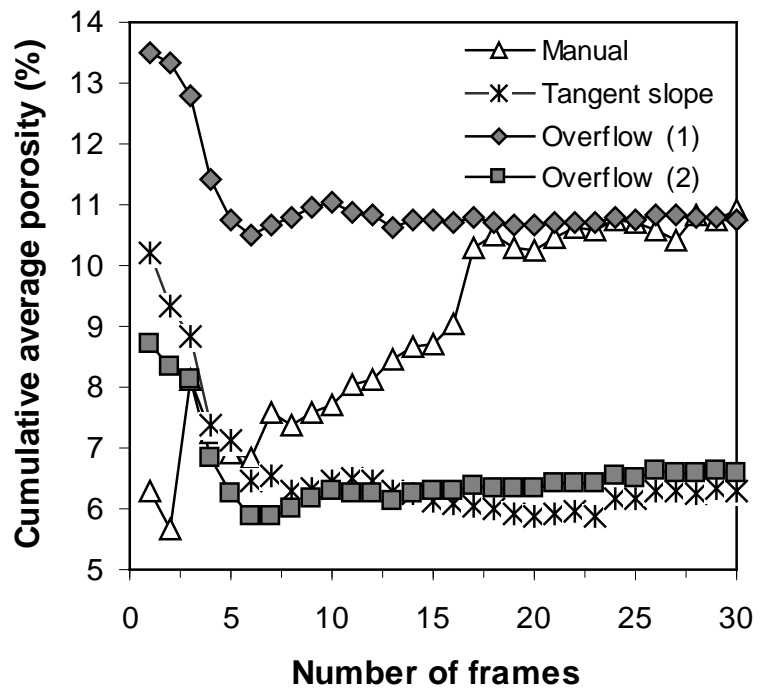

b)

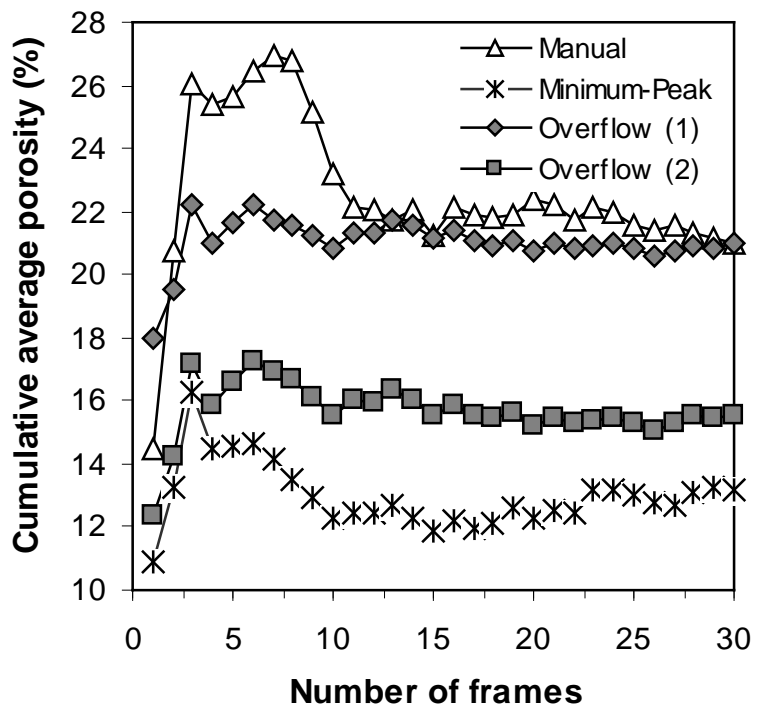

Fig. 7 Cumulative average porosity for a) Mortar A and b) Mortar B. The proposed segmentation method requires less number of frames to achieve a stable porosity value.

The mixtures investigated in this study represent very high and low porosity mortar, and the proposed technique performed well in both cases; therefore it should also be applicable to samples with intermediate porosity values. Although only one magnification level was investigated in this paper, albeit a commonly used one, unpublished 
observations show that the critical overflow point can also be found in the cumulative brightness histogram of images taken at 350x and 750x magnifications. Another advantage of this method is that it can be applied to a BSE image of an ordinary polished sample without requiring any special techniques to highlight the pores.

It is difficult, if not impossible to ascertain which segmentation method produces a more accurate porosity value. Possibly, by comparing results with other pore-quantification methods such as gas-adsorption or mercury porosimetry, an indication may be obtained, however, the fundamental differences and inherent weaknesses of these techniques must also be taken into account in making such comparisons. Another way is by correlating the image analysis porosity with some other physical characteristics such as mechanical strength and transport properties. Finally, it must be stressed that classification of pixels into their respective phases based on grey level can never be a perfect process, although as shown in this study, errors can be minimised by using an appropriate thresholding rule.

\section{Conclusion}

The feature-segmentation stage is of prime importance for accurate quantitative image analysis of microstructures. A good segmentation method is one that is autonomous and objective, returning an accurate and reproducible result. In this paper, a pore segmentation method for backscattered electron images of cement-based materials is presented. The method involves determining the porosity threshold from the inflection point of the cumulative brightness histogram of the grey level image. This value corresponds to the critical point where the segmented pore areas begin to 'overflow' to the surrounding paste. This technique was found to be more objective, reliable and economical than existing pore segmentation methods and resolves the difficulties in defining the true boundary of the pore phase, particularly for dense microstructures. The proposed segmentation method is able to produce a porosity value that is close to the one obtained by visual thresholding, but requires substantially fewer frames to achieve a statistically significant result. This technique facilitates future work on pore-structure characterisation via image analysis, which is an important subject in cement and concrete research since the pore phase controls not only strength and elastic properties, but also molecular transport properties and overall durability.

\section{Acknowledgements}

HSW would like to acknowledge the financial assistance provided by Universities UK, via the Overseas Research Students Awards Scheme. We thank Mr. R.A. Baxter for his help with the laboratory work. 


\section{References}

[1] K.L. Scrivener, P.L. Pratt (1984), Backscattered electron images of polished cement sections in the scanning electron microscope, Proc 6th Int Conf Cem Microscopy, Albuquerque, New Mexico, 145-155.

[2] P.J. Goodhew, J. Humphreys, R. Beanland (2001), Electron Microscopy and Analysis, Third edition, Taylor \& Francis, London, 251p.

[3] K.L. Scrivener (1987), The microstructure of anhydrous cement and its effect on hydration, Mat Res Soc Symp Proc, 85, 39-46.

[4] Y. Wang, S. Diamond (1995), An approach to quantitative image analysis for cement pastes, Mat Res Soc Symp Proc, 370, 23-32.

[5] K.L. Scrivener, H.H. Patel, P.L. Pratt, L.J. Parrott (1987), Analysis of phases in cement paste using backscattered electron images, methanol adsorption and thermogravimetric analysis, Mat Res Soc Symp Proc, 85, 67-76.

[6] K.L. Scrivener, E.M. Gartner (1988), Microstructural gradients in cement paste around aggregate particles, Mat Res Soc Symp Proc, 114, 77-86.

[7] K.L. Scrivener, A.K. Crumbie, P.L. Pratt, (1988) A study of the interfacial region between cement paste and aggregate in concrete, Mat Res Soc Symp Proc, 114, 87-88.

[8] D. Darwin, M.N. Abou-Zeid, K.W. Ketcham (1995), Automated crack identification for cement paste, Cem Concr Res, 25 (3) 605-616.

[9] D.A. Lange, H.M. Jennings, S.P. Shah (1994), Image analysis techniques for characterization of pore structure of cement-based materials, Cem Concr Res, 24 (5) 841-853.

[10] S. Diamond, M.E. Leeman (1995), Pore size distributions in hardened cement paste by SEM image analysis, Mat Res Soc Symp Proc, 370, 217-226.

[11] W. Reuter (1972), The ionization function and its application to the electron probe analysis of thin films, Proc. 6th Int. Conf. on X-Ray Optics and Microanalysis, Univ. Tokyo Press, Tokyo, 121.

[12] H. Zhao, D. Darvin (1992), Quantitative backscattered electron analysis of cement paste, Cem Concr Res, 22, 695-706.

[13] K.L. Scrivener (1989), The use of backscattered electron microscopy and image analysis to study the porosity of cement paste, Mat Res Soc Symp Proc, 137, 129-140. 
[14] J.C. Russ (2002), The Image Processing Handbook, Fourth edition, CRC Press, London, 744p.

[15] T. Pun (1980), A new method for grey-level picture thresholding using the entropy of the histogram, Signal Processing, 2, 223-237

[16] J.N. Kapur, P.K. Sahoo, A.K.C. Wong (1985), A new method for gray-level picture thresholding using the entropy of the histogram, Computer Vision, Graphics and Image Processing, 29, 273-285.

[17] N.R. Pal, S.K. Pal (1989), Entropic thresholding, Signal Processing, 16, 97-108.

[18] A. Ammouche, D. Breysse, H. Hornain, O. Didry, J. Marchand (2000), A new image analysis technique for the quantitative assessment of microcracks in cement-based materials, Cem Concr Res, 30, 25-35.

[19] A. Ammouche, J. Riss, D. Breysse, J. Marchand (2001), Image analysis for the automated study of microcracks in concrete, Cem Concr Compos, 23, 267-287.

[20] P. Soroushian, M. Elzafraney, A. Nossoni (2003), Specimen preparation and image processing and analysis techniques for automated quantification of concrete microcracks and voids, Cem Concr Res, 33, 1949-1962.

[21] J.I. Goldstein, D.E. Newbury, P. Echlin, D.C. Joy, C. Fiori, E. Lifshin (1981), Scanning Electron Microscopy and X-Ray Microanalysis. A Text for Biologists, Material Scientists, and Geologists, Plenum Press, New York, $673 p$.

[22] R. Yang, N.R. Buenfeld (2001), Binary segmentation of aggregate in SEM image analysis of concrete, Cem Concr Res, 31, 437-441.

[23] E.E. Underwood (1970), Quantitative Stereology, Addision-Wesley, Reading, Massachusetts, 274p.

\section{Appendix I}

The entropy maximisation method proposed by Kapur et al. [16] assumes that the entire histogram consists of two separate probability distributions; one for the object and the other for background. If $\mathrm{s}$ is the assumed threshold for the object, the entropy (information content) of the black portion (object) is

$$
H_{B} \quad=-\sum_{i=0}^{S} \frac{p_{i}}{P_{s}} \log _{2}\left(\frac{p_{i}}{P_{s}}\right)
$$

and for the white portion (background) is 


$$
H_{W} \quad=-\sum_{i=S+1}^{254} \frac{p_{i}}{\left(1-P_{s}\right)} \log _{2}\left(\frac{p_{i}}{1-P_{s}}\right)
$$

Where $P_{i} \quad=N_{i} / N$

$$
\begin{array}{ll}
N_{i} & =\text { number of pixels with brightness intensity } i \\
N & =\text { total pixels in the image } \\
P_{S} & =\sum_{i=0}^{S} p_{i}
\end{array}
$$

The total entropy of the image is

$$
H_{T} \quad=H_{B}+H_{W}
$$

The value $s$ that maximises $H_{T}$ is the threshold for object and background.

\section{Appendix II}

The number of frames required per sample, $N$, such that the sample mean $\bar{y}$ is within $\delta \%$ of the true population mean $\mu$, at 95\% degree of confidence can be calculated from Student's t-distribution:

$$
\begin{aligned}
t_{1-\alpha, v} & =\frac{\bar{y}-\mu}{S / \sqrt{N}} \\
\mathrm{~N} & =\left(\frac{t_{1-\alpha, v} \times S}{\bar{y}-\mu}\right)^{2} \\
& =\left(\frac{t_{1-\alpha, v} \times S \times 100}{\delta \times \mu}\right)^{2}
\end{aligned}
$$

If $S$ and $\mu$ is estimated from a sample size of 30, the critical t-value for a two-tailed test, $t_{0.025,29}$ is equal to 2.045. For $\delta$ $=10 \%$;

$$
\mathrm{N} \quad=\left(\frac{2.045 \times S}{0.1 \times \mu}\right)^{2}
$$




\section{Appendix III}

If $X_{i}$ and $Y_{i}$ represent pore and paste areas for frame $i$, then the average porosity $\mu$ after a total of $n$ frames, and the cumulative average porosity, $\mu_{n}$ is calculated based on the following:

$$
\begin{aligned}
\mu & =\frac{1}{n} \sum_{1}^{n}\left(\frac{X_{i}}{Y_{i}} \times 100\right) \\
\mu_{n} & =\left(\frac{\sum_{1}^{n} X_{i}}{\sum_{1}^{n} Y_{i}} \times 100\right)
\end{aligned}
$$

The average porosity and cumulative average porosity are only equivalent if the paste area for all frames is the same. 